\title{
LBBA: An Efficient Online Benefit-Aware Multiprocessor Scheduling for QoS via Online Choice of Approximation Algorithms
}

\author{
Behnaz Sanati $^{\dagger}$ and Albert M. K. Cheng \\ Real-Time Systems Laboratory, Computer Science Department \\ University of Houston, TX, USA \\ \{bsanati, cheng\}@cs.uh.edu
}

\begin{abstract}
Maximizing the benefit gained by soft real-time jobs in many applications and embedded systems is highly needed to provide an acceptable QoS (Quality of Service). This paper considers a benefit model for on-line preemptive multiprocessor scheduling. The goal is to maximize the total benefit gained by the jobs that meet their deadlines. This method prioritizes the jobs using their benefit density functions and schedules them in a real-time basis. We propose an online choice of two approximation algorithms in order to partition the jobs among identical processors at the time of their arrival without using any statistics. Our analysis and experiments show that we are able to maximize the gained benefit and decrease the computational complexity (compared to existing algorithms) while minimizing makespan (response time, also referred to as cost), with fewer missed deadlines and more balanced usage of processors. Our solution is applicable to a wide variety of soft real-time applications and embedded systems such as, but not limited to multimedia applications, medical monitoring systems or those with higher utilization such as bursty hosting servers. ${ }^{*}$
\end{abstract}

Keywords: Real-Time Scheduling; Multiprocessor; Embedded System; Partitioning; Load-Balancing;

\section{INTRODUCTION}

Multiprocessor platforms are widely adopted for many different applications in embedded systems and server farms. They are becoming even more popular since many chip makers including Intel and AMD are releasing multi-core chips. Adopting multiprocessor platforms can enhance the system performance, but scheduling jobs optimally on a multiprocessor system is an NP-hard problem [1], [2].

There are two major models for this scheduling problem. The first is the cost model; its goal is to minimize the cost, which is the overall flow time, also referred to as response time or makespan. The second model is the benefit model which aims to maximize the benefit of jobs that meet their deadlines. The latter model is used for soft real-time applications in which deadline misses are sometimes tolerable. Examples of such applications using multi-core platforms are multi-purpose home appliances such as HDTV streaming and interactive video games. More motivating examples from the domains of multimedia, air defense and enterprise-level, asynchronous, cooperating real-time computer systems are given by Welch and Brandt [3]. Other examples of such applications and embedded systems which use multi-core platforms are multimedia applications as explained in [4], image and speech processing [5], [6], [7], [8], time-dependent planning [9], robot control/navigation systems [10], [11], medical decision making [12], information gathering [13], real-time heuristic search [14], database query processing [15], and Internet of Things (IoT) [16].

In our research, we mainly focus on the benefit model to maximize the benefit of online preemptive scheduling of soft real-time jobs on multiprocessor systems having identical processors. In the meanwhile, we propose an online choice of two approximation algorithms, Greedy and Load-Balancing, to reduce the cost or makespan. More balanced distribution of the jobs between processors in our novel approach results in a lower overall flow time, less total idle time, fewer missed deadlines and more efficient usage of CPU cycles. Also, in our model, the NP hard problem of multiprocessor scheduling is reduced into uniprocessor scheduling problem by partitioning the tasks at their arrival time.

\footnotetext{
*This research is done by Behnaz Sanati and supervised by Professor Albert Cheng.

${ }^{\dagger}$ Corresponding author
}

(C) 2015. This manuscript version is made available under the Elsevier user license

http://www.elsevier.com/open-access/userlicense/1.0/ 
In the following subsections, we provide an overview of previous works on approximation algorithms and maximizing benefit on-line for multiprocessors. Section 2 presents the details of our new approach and an example to illustrate its differences from the previous methods. The analysis of the new method is provided in Section 3. Section 4 contains the performance metrics and our experimental settings and results. Finally the conclusions of this research are presented in Section 5.

\subsection{Related Work on Approximation Algorithms}

Approximation algorithms are often used to attack difficult optimization problems, such as job scheduling on multiprocessor systems which is an NP-hard problem [1], [17], [18]. An approximation algorithm settles for nonoptimal solutions found in polynomial time, when it is very unlikely to find an efficient exact algorithm to solve NP-hard problems, or the sizes of the data sets are so large that they make the polynomial exact algorithms too expensive.

A Greedy 2-approximation algorithm is used in [19] for fault tolerance and in [20] for benefit maximization in identical multiprocessor systems. Even though Greedy approximation can be a good solution in many cases, a load balancing approximation can result in a shorter flow time for a set of jobs when we have to distribute several jobs among multiple processors at the same time [21], [22]. Piel et al. [23] have proposed a load balancing technique based on statistics for real-time scheduling on asymmetric multiprocessors. They apply partitioning for high priority jobs and migration for jobs with low priority.

\subsection{Related Work on Benefit-Aware Real-Time Computing}

The gained benefit can vary when using different benefit functions. Researchers have investigated applying benefit functions for allocating resources in limited, soft real time systems [24], [25], [26]. Andrews et al. [27] provided a framework to formalize the use of benefit functions in complex real time systems.

Buttazzo et al. [28] provided the results of studying jobs that are characterized by an importance value. The performance of the scheduling algorithm was then evaluated by computing the cumulative value (or benefit) gained on a job set. However, the target of their research was uniprocessor scheduling. Welch et al. [3] discussed how benefit is used in a variety of real-time paradigms and in example applications. Awerbuch et al. presented a constant competitive ratio algorithm for a benefit model of on-line preemptive scheduling [29]. This method can be used on both uniprocessor and multiprocessor systems. Aydin et al. [30] proposed a reward-based scheduling method for periodic real-time tasks and [31] presented on-line scheduling policies for a class of IRIS (Increasing Reward with Increasing Service) real-time tasks.

\section{OUR CONTRIBUTION: ONLINE CHOICE OF APPROXIMATION ALGORITHMS}

The algorithm proposed in [29] only focuses on maximizing the total benefit gained without being concerned with minimizing the overall flow time of a job set (response time or makespan). In the meanwhile, in that method, the benefit gained by each job that completes its execution is calculated using the benefit density function of its flow time. This function is a non-increasing, non-negative function of time, by definition [29]. It means the more the flow time, the less the benefit gained.

Therefore, we proposed, simulated and analyzed an efficient online benefit-aware technique with choices of approximation algorithms including Greedy and load balancing to partition jobs among multiple processors at the time of release. This method prioritizes the jobs using their benefit density functions and schedules them in a realtime basis in order to reduce the makespan (overall flow time) of the jobs and total idle time of the processors while maximizing the total gained benefit.

We also used our online choice of two approximation algorithms (Greedy and Load-Balancing) as a solution for special cases that were not considered in the existing benefit-aware multiprocessor scheduling algorithms such as the Benefit-Based Algorithm proposed in [29] which we refer to as BBA in the rest of this paper. Examples of those cases are when there are several high priority jobs which can preempt a running job or when a high priority job can preempt more than one running job.

In order to be able to balance the workload among the processors by partitioning the jobs as soon as they are released, we consider a separate pool of the waiting jobs for each processor. Our method is referred to as LoadBalancing/Greedy Benefit-Aware algorithm (or LBBA) throughout the paper. This load balancing technique is different from what Piel et al. [23] have used, since we do not use statistics for distributing the jobs. Instead, we make decisions online by using the actual (worst case) execution times of ready jobs and the remaining workload of the processors for partitioning on a real-time system with identical processors. Migration is not allowed in our real-time system model. 


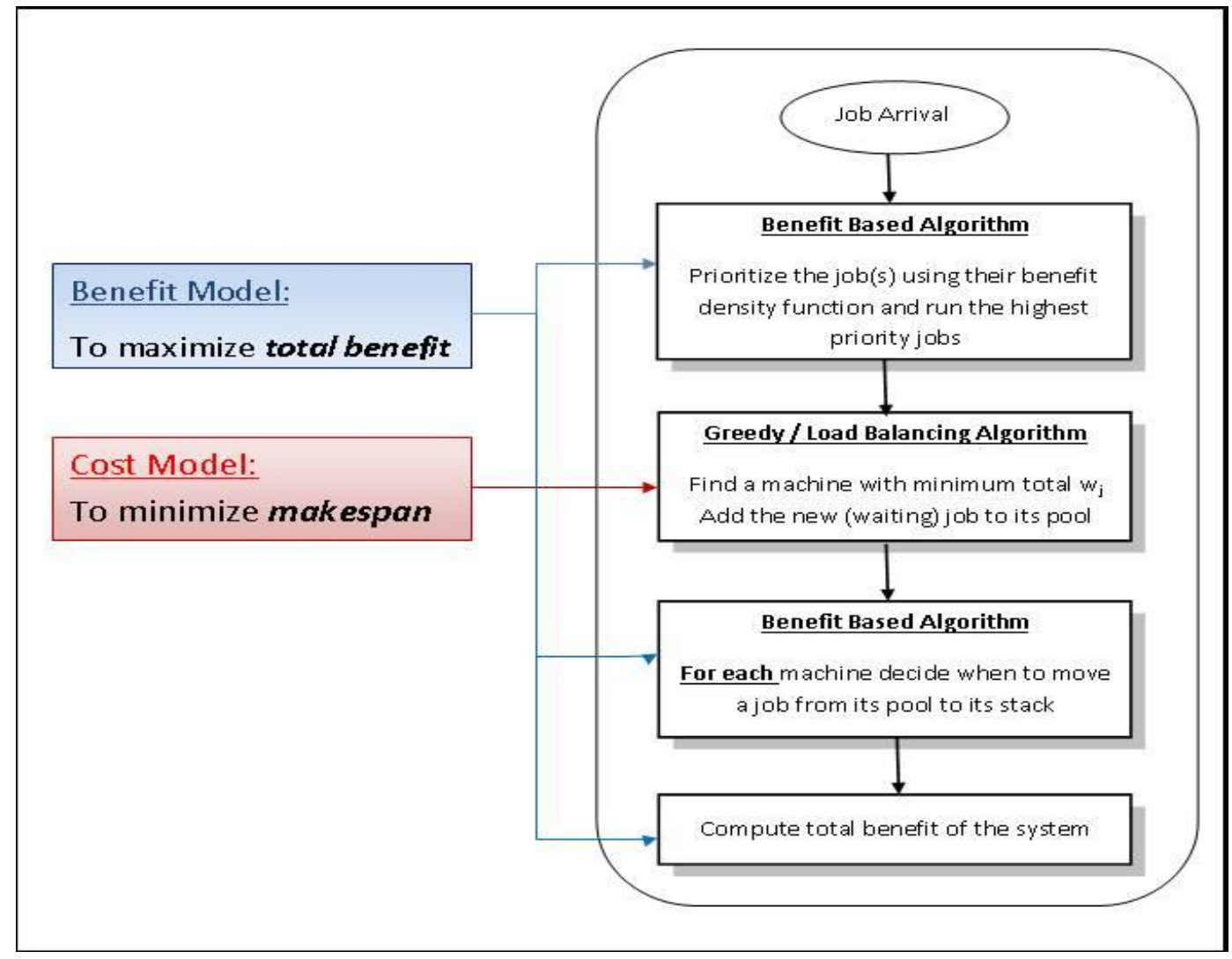

Fig.1. LBBA Methodology

LBBA is superior to BBA in principle, since:

- $\quad$ LBBA is a novel hybrid model of soft real-time multiprocessor scheduling. Despite of BBA, which only follows benefit model, LBBA is a combination of benefit model and cost model. That is, it aims to minimize makespan in order to achieve the maximum benefit at the lowest cost.

- Reducing multi-processor scheduling into uniprocessor scheduling: BBA uses one pool shared by all the processors to keep the ready jobs while they're not scheduled. Hence, partitioning of each job is done when it is scheduled, and starts running on the assigned processor. Considering a separate pool for each processor, enables the LBBA scheduler to partition the jobs at their arrival time. The advantage of this method of early partitioning is that the NP-Hard problem of multiprocessor real-time scheduling will be reduced to a series of uniprocessor realtime scheduling.

- LBBA facilitates load-balanced partitioning of waiting jobs, while this case is not considered in BBA. For example, in case the waiting (or ready) jobs arrive asynchronously, then LBBA adapt the "Greedy Approximation" to assign a job to the pool of the processor with the least remaining workload. If jobs are synchronous, i.e. arrive at the same time, those that cannot start running and have to wait in a pool, will be partitioned among the processors, using our "Load-Balancing" technique.

- $\quad$ LBBA optimizes the CPU usage and minimizes the total idle time of the processors by balancing the workload among them.

- It improves Quality-of-Service (QoS) by reducing missed deadline ratio: As shown by an example in 2.4, LBBA reduces the possibility of starvation for low priority jobs, comparing to BBA. It also has Minimal Response time, including both scheduling and execution time, for a job set (up to $300 \%$ faster response time than BBA in our experiments shown in 4.1.2.).

- $\quad$ LBBA is computationally less expensive than BBA, as we prove in sub-section 3.2. 
The reason for which we are applying both Greedy and Load-Balancing approximation algorithms, depending on the situation, instead of only one of them all the time, are as follows:

\section{A. Why we do not use Greedy approximation all the time:}

The Greedy-Benefit-Based model, called GBBA, that we proposed in [20] in early stage of this research handles the special cases we mentioned earlier, that were overlooked by BBA, by applying a 2-approximation Greedy method. However, it has a shortcoming in minimizing makespan when several ready jobs are going to be partitioned among the pools of the processors at the same time. Also, this is the case when several synchronous high priority jobs can preempt more than one running job. To overcome this problem, we add our Load-Balancing approximation method to GBBA. Our hypothesis is that the online combination of these two approximation methods can minimize the makespan while maximizing the total benefit.

\section{B. Why we do not use Load-Balancing all the time:}

In Load-Balancing, we sort the jobs in descending order of their workload and the processors in ascending order of their total remaining workload (both on the stack and the pool of each processor). Then the first job in the TempList (list of the ready jobs) which has the heaviest workload, will be assigned to the first processor in the list, having the least remaining workload. Second job in the list will be assigned to the second processor and so on.

This method, by itself and without the help of Greedy approximation, can be used to partition the jobs among the processors so that the distributed workload is as balanced as possible. Greedy approximation does not facilitate Load-Balancing, when many-to-many assignments are needed. However, in order to optimize computation time, we use Greedy method in cases that a one-to-many or many-to-one assignment is needed.

In this research, both BBA and our solution, LBBA, are simulated extensively in order to compare their performances. Figure 1 demonstrates LBBA hybrid methodology which is a combination of benefit model and cost model. In the following sub-sections, we define our system and task model, and the notations used in our algorithm, along with the detailed explanation of LBBA algorithm. At the end of this section, we illustrate the advantages of LBBA over BBA and GBBA through an example.

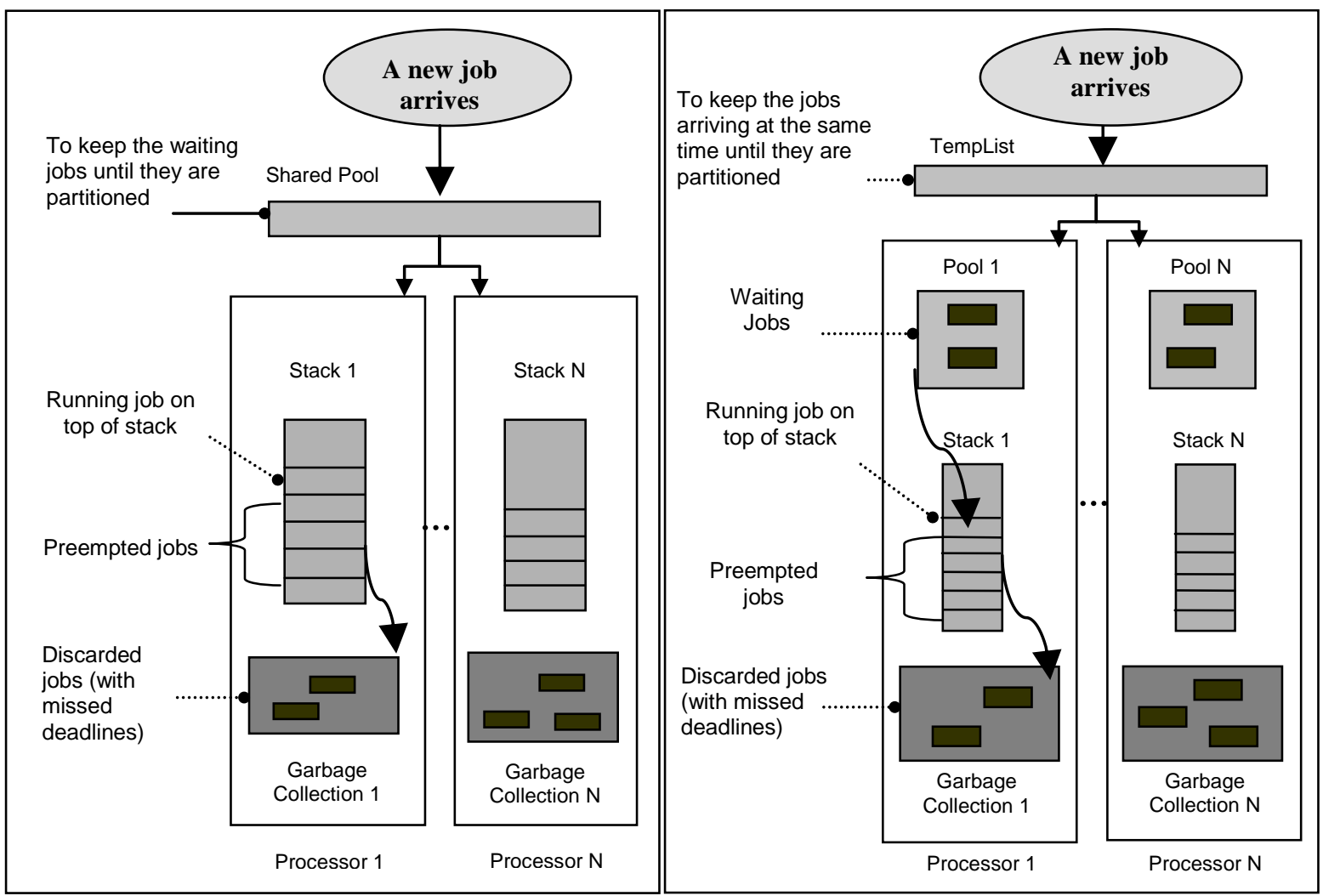

Fig.2. Job storage locations with a shared pool in BBA (left) and a pool for each processor in LBBA (right) 


\subsection{System and Job Model}

A multiprocessor system with $m$ identical processors is considered for our partitioning approach. In the partitioning approach no migration of jobs is allowed. Therefore, each job has to stay with only one processor during its whole execution time. This method is possible if each processor has its own pool instead of sharing a pool with other processors. Also, as Figure 2 shows, each processor has its own stack and garbage collection. This paper explores the scheduling of aperiodic soft real-time job sets which are independent in execution and there are no precedence constraints among them. Pre-emption is allowed. Each aperiodic job may be released at any time. An example of such aperiodic jobs is a partial air defense subsystem also mentioned by Welch et al. [3].

\subsection{Definition of Notations}

We define the notations that are used throughout this paper as follows:

$r_{j}$-release time of job $J_{j}$

$w_{j}$-execution time of job $J_{j}$, simply considered as workload of job $J_{j}$ in this paper

$s_{j}-$ start time of job $J_{j}$

$c_{j}$ - completion time of job $J_{j}$

$B r_{j}$-break point or deadline of job $J_{j}$,

$$
B r_{j}=s_{j}+2 w_{j}
$$

$\beta_{j}(t)$ - benefit density function of job $J_{j}$ at time $t$, for $\left(t \geq w_{j}\right)$, which is a non-increasing, non-negative function, with the following restriction to be satisfied for each $\beta_{j}(t)$ :

$$
\frac{\beta_{j}}{\beta_{j}\left(t+w_{j}\right)} \leq C
$$

Note: for $t<w_{j}$, there would be no benefit gained by job $J_{j}$, since it has certainly not completed its execution at time $t$.

$f_{j}$-flow time of job $J_{j}$ :

$b_{j}$ - benefit, gained by a completed job $J_{j}$ :

$$
f_{j}=c_{j}-r_{j}
$$

$$
b_{j}=w_{j} \cdot \beta_{j}\left(f_{j}\right)
$$

$d_{j}(t)$ - variable priority of job $J_{j}$ at time $t$, before scheduling $\left(t<s_{j}\right)$ :

$$
d_{j}(t)=\beta_{j}\left(t+w_{j}-r_{j}\right)
$$

$d_{j}^{\prime}-$ fixed priority of job $J_{j}$, when it is scheduled and starts its execution:

$$
d_{j}^{\prime}=\beta_{j}\left(s_{j}+w_{j}-r_{j}\right)
$$

\subsection{Our Algorithm}

We are adopting the same definition of breakpoint, benefit density function, priority of the jobs in pools or on the stacks as used in BBA, and also the same preemption condition to be able to determine if we could improve that algorithm by applying approximation algorithms and necessary modifications to the system. However, to show that our solution does not sacrifice benefit maximization in order to obtain the minimum response time, we will prove that the competitiveness of BBA is also preserved in our algorithm.

A desired property of the system in this method is the possibility to delay jobs without drastically reducing overall system performance. Also, this algorithm does not use migration on the multiprocessor system. The LBBA algorithm is an event-driven algorithm. The events are new job arrival, job completion, and reaching the break point of a job. The algorithm takes action when a new job arrives, a running job completes, or when a running job reaches its break point. When new jobs arrive they will be partitioned among the processors.

Each job $j$ arrives with its own execution time $\left(w_{j}\right)$ and benefit density function $B_{j}(t)$ for $\left(t \geq w_{j}\right)$. The flow time of a job, denoted by $f_{j}$, is the time that passes from its release time $\left(r_{j}\right)$, to its completion time $\left(c_{j}\right)$ and is at least equal to $w_{j}$ (execution time). The benefit gained by each job that completes its execution is a function of its flow time (equation 4). The job on top of each stack is the job that is running and all other jobs in the stacks are preempted. If a job reaches its breakpoint and its execution is not completed yet, it will not be able to gain any benefit; therefore, it will be popped from the stack and sent to the garbage collection. This means the break point of a job is its deadline, which is twice its execution time after it starts running.

The priority of each unscheduled job (located in each pool) at time $t$ which is denoted by $d_{j}(t)$ (for $t \leq s_{j}$ ) is variable with time. However, for $t>s_{k-}$ (when the job $k$ has started its execution) the priority is calculated as $d^{\prime}{ }_{k}$ $=B_{k}\left(s_{k}+w_{k}-r_{k}\right)$ (lines 19 and 68 of the following pseudo-code). The notation $d^{\prime}{ }_{k}$ is used for the priority of the 
running job $k$ on top of the stack. This priority is given to the job $k$ when it starts its execution. Its start time, $s_{k}$, is used in the function instead of variable $t$, therefore its priority is no longer dependent on time. Since $s_{k}$, $w_{k}$ and $r_{k}$ are all fixed values, the priority of a job will not change after its start time.

Once a new job $j$ is released, if there is a processor such that $d_{j}(t)>4 d^{\prime}{ }_{k}$ (lines 58 through 66 ), or its stack is empty (lines 11 through 22), then the newly released job is pushed onto the stack and starts running, otherwise it will be partitioned among the pools of the processors using an online choice of load balancing or Greedy approximation (lines 39 through 75). Awerbuch et al. [29] used the preemption condition $\left(d_{j}(t)>4 d^{\prime}{ }_{k}\right)$ and their analysis shows that the factor 4 in this condition plays role in the BBA constant ratio competitiveness being equal to $10 C^{2}$. Therefore, in order to preserve this competitiveness, we use the same criteria. Later in the analysis of our algorithm, we prove how this competitiveness is preserved by LBBA.

When a currently running job on a processor completes, it is popped from the stack. Then, the processor runs the next job on its stack if $d_{j}(t) \leq 4 d^{\prime}{ }_{k}$ for all $j$ in its pool, otherwise, it gets the job with max $d_{j}(t)$ from its pool, pushes it onto the stack and runs it. The completed jobs or those that reach their break points are going to be sent to the garbage collection. If a job completes before reaching its break point, its gained benefit is calculated and added to the total benefit. If more than one high priority job is able to preempt some running job(s), to decide which job should be sent to which stack, we send the largest job to the processor with the minimum remaining work load, the second largest job to the processor with the second smallest remaining work load, so on so forth. This way we are able to balance the work load among the processors. However, in case there is only one high priority job at a time instance which can preempt more than one running job, we assign it to the stack of the processor with minimum remaining execution time (Greedy approximation).

To be able to assess the performance of LBBA, we need to consider various situations of the released jobs, regarding their release times and workloads. Here, we discuss different scenarios and how they are handled. In addition, we provide an example in which BBA can result in very long waiting time for some jobs before they get scheduled or even their starvation. We show that LBBA overcomes this problem and that is one of the key aspects of LBBA which reduces the missed deadline ratio and improves the Quality of Service.

\section{Case $1: J_{j}$ is a newly released job at time $t$}

Lemma 1: For a newly released job, $J_{j}$, at time $t$, its priority is independent of its release time, $r_{j}$, but relies on its workload, $w_{j}$.

Proof: Since $J_{j}$ is released at time $t$,

From the equations (5) and (7): $\quad \begin{aligned} t & =r_{j} \\ d_{j}\left(r_{j}\right) & =B_{j}\left(w_{j}\right)\end{aligned}$

So, Lemma 1 is proved. Equation (8) shows that the priority of job $J_{j}$ at its release time is a function of $w_{j}$ regardless of its release time, $r_{j}$.

Case $2: J_{i}$ is a waiting job in a pool

Lemma 2: If $J_{j}$ cannot start its execution at its release time, it has to wait in pool, then its variable priority $d_{j}(t)$ will not increase at any time $t,\left(r_{j}<t<s_{j}\right)$ while it is waiting.

Proof: At any time instance $t$, while $J_{j}$ is waiting $t>r_{j}$. By definition, $d_{j}(t)=B_{j}\left(t+w_{j}-r_{j}\right)$ and also $B_{j}$ is a nonincreasing, non-negative function. Hence, Lemma 2 is proved:

For all $t, t>r_{j}, \quad \quad d_{j}(t) \leq d_{j}\left(r_{j}\right)$

Theorem 1: If $J_{i}$ is released and cannot be scheduled at release time by BBA, if the next jobs have the same workload as $J_{i}$ or less, $J_{i}$ may starve or wait until all of them are scheduled.

Proof: Based on Lemma 1 and Lemma 2, if $J_{i}$ is waiting in the shared pool (in BBA method) when $J_{j}$ is released, then at $\mathrm{t} \geq r_{j}$, its priority will be less than the priority of $J_{j}$. So, if any processor is available or the priority of $J_{j}$ is high enough to preempt another job, $J_{j}$ is scheduled before $J_{i}$. If the next released jobs, all have the same or smaller workload than $J_{i}$, then it has to wait in the shared pool until all of them are scheduled.

In LBBA, $J_{i}$ instead of being kept in the shared pool, will be assigned to the pool of a processor based on Greedy or Load-Balancing method, depending on the situation. Also, the next released jobs will not all get assigned to the same pool and will be distributed among all processors. This means the waiting time of $J_{i}$ will be significantly less in LBBA than BBA method. 
A summarized version of the algorithm is as follows:

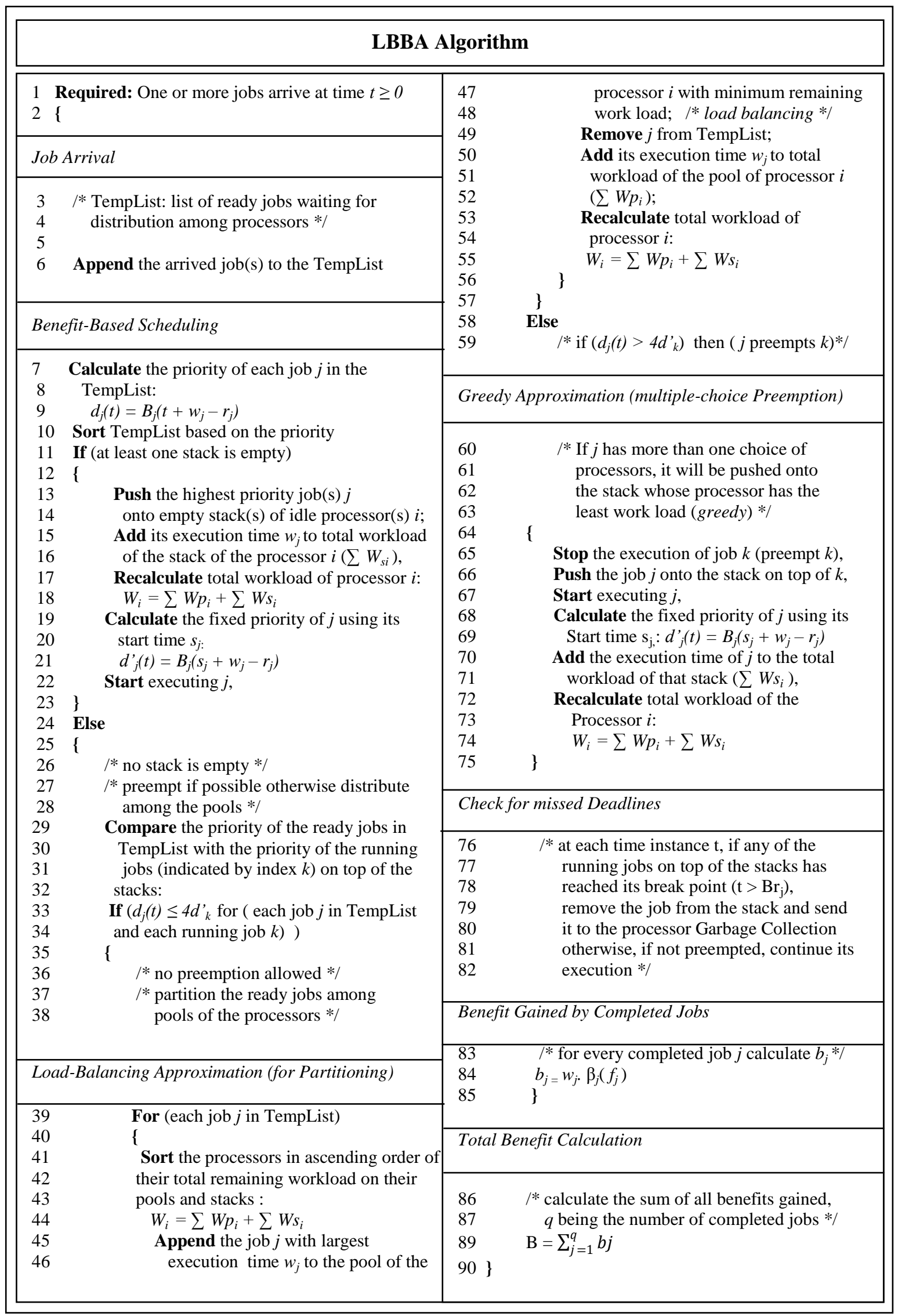


Theorem 2: If $J_{j}$ cannot preempt any currently running jobs at its arrival (i.e. release time), then it will not be able to preempt any jobs that start running after release of $J_{j}$ while $J_{j}$ is waiting.

Proof: There will be two different scenarios for this situation. Hence, Theorem 2 can be deduced from the two following Lemmas.

Lemma 3: Let $J_{j}$ be a job that is waiting in a pool. If $J_{s}$ is released after $J_{j}\left(r_{s}>r_{j}\right)$, and it is scheduled before $J_{j}$, then it cannot be preempted by $J_{j}$ any time during its execution.

Proof: $J_{s}$ is released at $r_{s}$, after release of $J_{j}$ (i.e. $r_{\mathrm{s}}>r_{j}$ ); $J_{s}$ is scheduled and starts its execution, while $J_{j}$ is waiting at $s_{s}$ (start time of $J_{s}$ ), therefore,

At $t_{l}=s_{s}$

$$
\begin{aligned}
& d^{\prime}{ }_{s}>d_{j}\left(t_{1}\right) \\
& d^{\prime}>d_{j}\left(s_{s}\right)
\end{aligned}
$$

Based on the priority assignment rule of the algorithm, $d_{s}{ }_{s}$, the priority of $J_{s}$ at time $s_{s}$ is a fixed priority and will not change with time, for $t \geq s_{s}$. However, the priority of $J_{j}$ which is still waiting, will not increase:
For $t_{2} \geq t_{1}$,
$d_{j}\left(t_{2}\right) \leq d_{j}\left(t_{1}\right)$
$d_{j}\left(t_{2}\right)<d_{j}\left(s_{s}\right)$
From 8 and 10:

$$
d_{j}\left(t_{2}\right)<d_{s}^{\prime}
$$

Hence, $J_{j}$ will not be able to preempt $J_{S}$ at any time after $J_{S}$ starts running, due to its priority not being high enough to preempt $J_{S}$.

Lemma 4: If $J_{j}$ is a waiting job, it cannot preempt any running job $J_{p}\left(r_{p} \leq r_{j}\right)$ and was scheduled either before $J_{j}$ was released $\left(r_{p}<s_{p}<r_{j}\right)$ or when $J_{j}$ was released $\left(r_{p} \leq r_{j} \leq s_{p}\right)$.

Proof: From Lemma 1, if $J_{j}$ cannot preempt $J_{p}$ at $t=r_{j}$, assuming $J_{p}$ was running at $r_{j}$, it will not preempt $J_{p}$ at any time $t>r_{j}$, since $d_{p}^{\prime}>d_{j}$ for all time instances $t \geq r_{j}$.

Also, if $J_{p}$ was released at the same time or before $J_{j}$ was released at $t=r_{j}$, and $J_{p}$ is scheduled before $J_{j}\left(r_{p} \leq\right.$ $r_{j} \leq s_{p}$ ), it shows that:

at $t=s_{p}$,

$$
\begin{aligned}
& d_{p}(t)>d_{j}(t) \\
& d^{\prime}{ }_{p}=d_{p}\left(s_{p}\right) \\
& d^{\prime}{ }_{p}>d_{j}\left(s_{p}\right) \\
& d_{j}(t) \leq d_{j}\left(s_{p}\right) \\
& d_{j}(t) \leq d^{\prime}{ }_{p}
\end{aligned}
$$

Therefore,

Hence, for $t>s_{p}$ :

From (12):

From (17) and (18):

\begin{tabular}{|c|c|c|c|c|c|}
\hline \multirow{2}{*}{ Job ID } & \multirow{2}{*}{$\begin{array}{c}\text { Arrival } \\
\text { Time }\left(r_{j}\right)\end{array}$} & \multirow{2}{*}{$\begin{array}{c}\text { Execution } \\
\text { Time } \\
\left(w_{j}\right)\end{array}$} & \multicolumn{3}{|c|}{ Benefit Gained by } \\
\hline & & & BBA & GBBA & LBBA \\
\hline$a$ & 0 & 8 & 0.00 & 0.00 & 0.33 \\
\hline$b$ & 1 & 4 & 0.40 & 0.40 & 0.40 \\
\hline$c$ & 1 & 6 & 0.50 & 0.43 & 0.43 \\
\hline$d$ & 2 & 3 & 0.21 & 0.25 & 0.25 \\
\hline$e$ & 3 & 2 & 0.50 & 0.50 & 0.50 \\
\hline$f$ & 3 & 4 & 0.25 & 0.22 & 0.15 \\
\hline$g$ & 3 & 6 & 0.21 & 0.27 & 0.27 \\
\hline$h$ & 6 & 2 & 0.50 & 0.50 & 0.50 \\
\hline$i$ & 6 & 1 & 0.50 & 0.50 & 0.50 \\
\hline$j$ & 8 & 2 & 0.50 & 0.50 & 0.50 \\
\hline$k$ & 9 & 3 & 0.50 & 0.25 & 0.25 \\
\hline$l$ & 10 & 2 & 0.50 & 0.50 & 0.50 \\
\hline
\end{tabular}

So, $J_{j}$ will not be able to preempt $J_{S}$ in this case and Lemma 4 is proved.

\subsection{An Example}

The following example, provided in Table 1, is a set of independent, real-time jobs which contains both synchronous and asynchronous jobs. The WCET (Worst-Case Execution Time) of each job will be known when it arrives. Figure 3 shows how this job set will be scheduled by BBA, GBBA and LBBA on a 3-processor system. The benefit gained by each completed job is calculated using $\beta_{j}(t)=1 /\left(2 w_{j}\right)$ as the benefit density function, and shown in Table 1 :

Table 1: An Example of a Job Set and job benefits gained by BBA, GBBA and LBBA 
a) $\underline{\text { BBA }}$

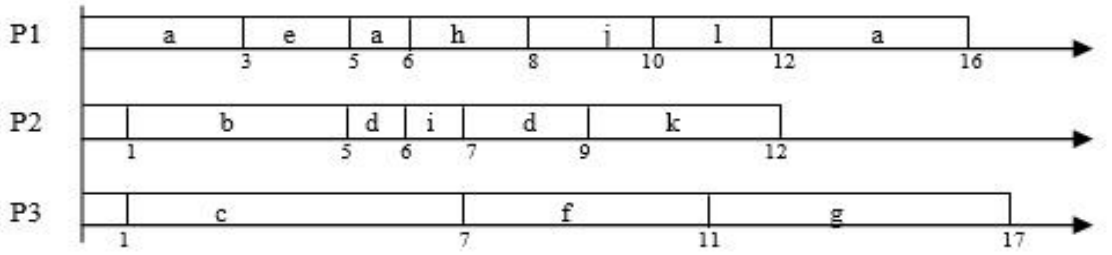

b) GBBA

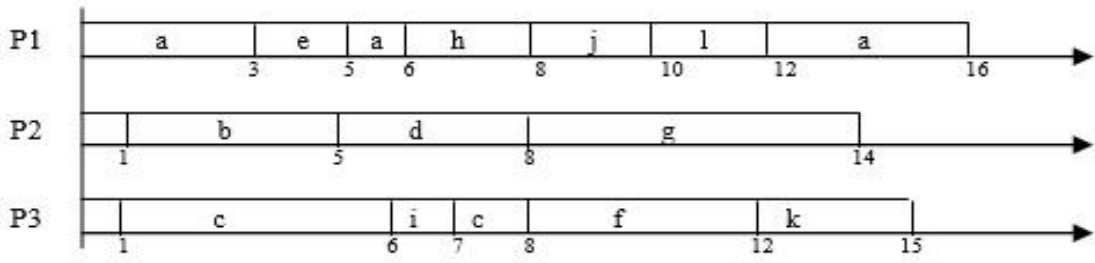

c) $\underline{\text { BBBA }}$

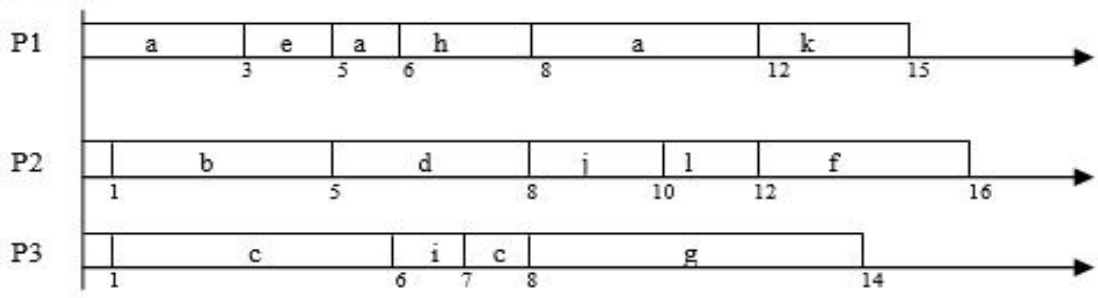

Fig.3. Job scheduling on a 3 processor system by BBA, GBBA and LBBA

\begin{tabular}{|c|c|c|c|c|c|c|}
\hline Algorithm & $\begin{array}{c}\text { Missed } \\
\text { Jobs }\end{array}$ & Preemptions & MakeSpan & $\begin{array}{c}\text { Total Idle } \\
\text { Time }\end{array}$ & $\begin{array}{c}\text { Total } \\
\text { Benefit }\end{array}$ & $\begin{array}{c}\text { Benefit-to-Cost } \\
\text { Ratio }\end{array}$ \\
\hline BBA & 1 & 5 & 17 & 8 & 4.57 & 0.269 \\
\hline GBBA & 1 & 5 & 16 & 5 & 4.32 & 0.270 \\
\hline LBBA & $\mathbf{0}$ & $\mathbf{3}$ & $\mathbf{1 6}$ & $\mathbf{5}$ & $\mathbf{4 . 5 8}$ & $\mathbf{0 . 2 8 6}$ \\
\hline
\end{tabular}

Table 2: Comparing the Performance of BBA, GBBA and LBBA

We can summarize the performance of the three scheduling methods considering the following metrics:

- Preemptions: When job $a$ is scheduled by BBA and GBBA schedulers, it gets preempted twice by higher priority jobs $e$ and $h$, also kept in preemption by jobs $j$ and $l$. It completes its execution right at its break point which is at time $t=16$. Therefore, job $a$ does not gain any benefit, even though the system has fully executed it, and it is considered as a missed job in both BBA and GBBA. However, it is only preempted by jobs $e$ and $h$ under LBBA scheduling. Job $i$ preempt job $d$, in BBA, while it preempt job $c$ in both GBBA and LBBA.

- Makespan: LBBA and GBBA have shorter makespan than BBA.

- Total Benefit: LBBA not only preserves the benefit maximization aspect of BBA, but also exceeds that. Also, it improves GBBA in this regard.

- Total Idle Time: In LBBA, total idle time was $38 \%$ less than BBA and GBBA.

- Benefit-to-Cost Ratio: As shown in the Table 2, benefit-to-cost ratio of LBBA is higher than BBA and GBBA and it is improved about $6.3 \%$ in this example.

- Missed Ratio: The ratio of missed jobs in BBA is $8.33 \%$ while in GBBA and LBBA was $0.00 \%$.

This example demonstrates how LBBA can improve the QoS, comparing two other state of the art benefitaware methods, BBA and GBBA, by: 
- Maximizing total benefit

- Maximizing benefit-to-cost ratio

- Minimizing total idle time

- Minimizing makespan

- Minimizing missed ratio

\section{ANALYSIS}

In order to evaluate our proposed algorithm, we analyze it from two points of view: computational complexity and benefit maximization.

\subsection{Job Benefit Maximization}

BBA is proved in [29] to be a constant competitive ratio algorithm $\left(10 C^{2}\right)$ for both uniprocessor and multiprocessor scheduling. This is with considering the restriction shown in equation (2), $\beta_{j} / \beta_{j}\left(t+w_{j}\right) \leq C$, to be satisfied for each $\beta_{j}(t)$ and some fixed constant $C$. That is, in case of delaying a job by its length, we only lose a constant factor in its benefit.

In order to preserve the competitiveness of that algorithm, we are adopting the same definition of breakpoint, benefit density function and its restriction, priority setting for the jobs in the pools or on the stacks, and also the same preemption condition. Therefore, the same proof of that competitiveness $\left(10 C^{2}\right)$ is true for each processor the same way as for BBA uniprocessor scheduling. That is because after partitioning, no migration is allowed and we have uniprocessor scheduling for the set of jobs on the pool of each processor based on the priority of the jobs, also the running job on each processor can be preempted by a newly arrived high priority job (in TempList) if the preemption condition is satisfied.

If $m$ denotes the number of processors, $i$, the index of a processor, $v_{i}^{O P T}$, total benefit of each processor by optimal scheduler and $v_{i}^{L B B A}$, total benefit of each processor by LBBA:

$$
v_{i}^{L B B A} \geq \frac{1}{10 C^{2}} v_{i}^{O P T}
$$

Then, adding up the benefits gained by all the processors in the system will result in:

$$
\sum_{i=1}^{m} v_{i}^{L B B A} \geq \frac{1}{10 C^{2}} \sum_{i=1}^{m} v_{i}^{O P T}
$$

Now, let $V^{O P T}$ denote total benefit gained by the optimum scheduling of a set of jobs and $V^{L B B A}$ total benefit gained by LBBA for the same job set. Therefore, equation (22) shows that algorithm LBBA is also $10 C^{2}$ competitive:

$$
V^{L B B A} \geq \frac{1}{10 C^{2}} V^{O P T}
$$

\subsection{Computational Complexity}

In the BBA method, at each time step, the priority of all jobs in the shared pool must be compared with the priority of the running jobs on the top of all processor stacks. If there are $m$ processors in the system and $n$ waiting jobs in the pool, $n$ times $m$ comparisons are done at each time step to determine if any of the waiting jobs can be pushed onto any stack and start running.

On the other hand, our method performs $(m-1)$ comparisons at each job arrival to find the least utilized processor and adds the execution time of new job $j$ to its utilization for future comparisons, resulting in $m$ operations at each job arrival. However, if $r$ jobs arrive at the same time $(r>1)$, a load-balanced/Greedy partitioning is performed: The jobs will be sorted based on a non-increasing order of their execution times, which roughly needs $r \log _{2} r$ comparisons. Then, the first job in the list is assigned to the pool of the processor with the least remaining workload, so on so forth.

At each time step, if $x_{1}$ is the number of waiting jobs in first pool, $x_{2}$ in the second pool, and so on so forth, then $X$ denotes the total number of waiting jobs $\left(X=x_{1}+x_{2}+\ldots+x_{m}\right)$. Since our new method only compares the priorities of waiting jobs in each pool with the priority of the running job on the corresponding stack, only $X$ comparisons are done at each time step. It is now clear that our method is computationally less expensive than the original one.

In the next section, we provide the results of our extensive experiments which show a significant improvement in the scheduling speed by our method especially for systems with very large work load. 


\section{PERFORMANCE EVALUATION}

Schedulability is one of the main performance metrics to evaluate a scheduler for hard real-time systems. However, benefit-aware schedulers are mainly used for soft real-time systems, in which missing a deadline would not drastically affect the performance. For soft real-time scheduling, total value or benefit gained and also miss ratio are the performance metrics. In this research, we considered the following measurements to evaluate and compare the performance of both BBA and LBBA algorithms:

- $\quad$ The benefit gained by completed jobs

- $\quad$ Missed deadline ratio

- $\quad$ The benefit-to-cost ratio

- Total processor idle time

The cost is the overall flow time of a job set. It is the time that has passed since the first job has arrived till the last job is completed. The benefit-to-cost ratio is calculated by dividing the total benefit by the overall flow time.

Also, reducing flow time and using processors more efficiently are other goals addressed by our solution. Therefore, to measure how efficiently CPU cycles have been used by either algorithm, we have considered total processor idle time. Total processor idle time is obtained by accumulating all the time periods in which any of the processors has been idle. The more the total idle time decreases while preserving or even improving the amount of gained benefit, the more efficiently processors are used and the shorter the response time or overall flow time (or cost) gets.

\subsection{Experimental Settings and Results}

In order to evaluate the performance of our method and compare it with the performance of the previous one, we implemented both algorithms in $\mathrm{C}++$ to simulate the scheduling of synthetic job sets using different numbers of identical processors.

For benefit density functions of the jobs, we tried different non-negative, non-increasing functions such as $B(x)=\frac{1}{n x}, B(x)=\frac{n}{x}$ and so on; where $x$ and $n$ were both positive integer numbers.

Also, we generated hundreds of job sets with randomly generated numbers as their arrival times and execution times, using the Poisson distribution which is applied by operations research to model random arrival times, especially for systems using queues, such as web servers and print servers.

\subsubsection{Synthetic Job Sets}

This experiment was done using aperiodic job sets. Each aperiodic job arrives with its own benefit density function and execution time. The arrival time and execution time of the jobs were randomly generated. Both algorithms were tested by scheduling hundreds of job sets with $20,40,60,80$, and 100 jobs which were randomly generated using a Poisson distribution. It is a discrete probability distribution that expresses the probability of a given number of events occurring in a fixed interval of time and/or space if these events occur with a known average rate and independently of the time since the last event. This is the same attribute we have considered for the job sets, being independent and aperiodic.We used the range of $[0,10]$ for their arrival times and $[1,15]$ for their execution times. We simulated the scheduling of the job sets for the systems having 2 and 3 identical processors.

Also, we tested both algorithms by scheduling sets of 50,100,150, 200, and 250 jobs for the systems having 4 and 8 identical processors. Their arrival times range was $[0,10]$ and their execution times range was $[1,20]$. We considered a shorter range for the arrival times comparing to the range of their execution times to simulate systems with heavier workloads comparing to the job sets generated for 2 and 3 processor systems.

\subsubsection{Experimental Results and Discussion}

The comparison of the results for BBA and LBBA are shown in separate graphs for each performance measurement and different number of processors (Figure 3). The improvement (or increase) in the total benefit gained by LBBA, ranged from $6.19 \%$ to $9.0 \%$ for a 2 processor system and from $5.04 \%$ to $7.10 \%$ for a 3 processor system (Figure 3.e and 3.f). 


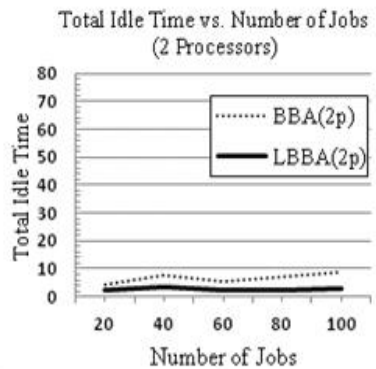

a)

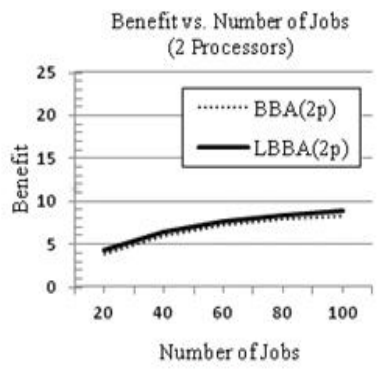

e)

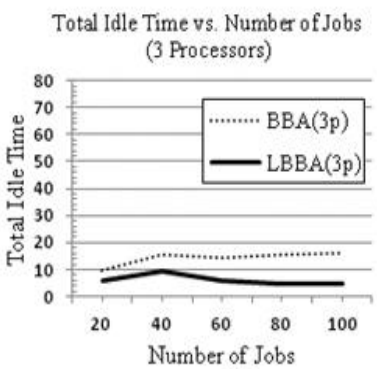

b)

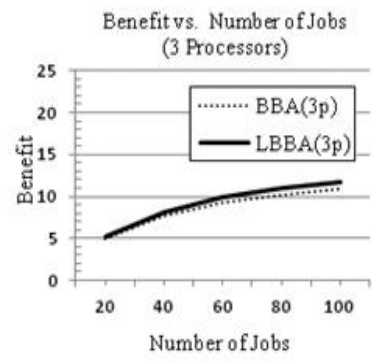

f)

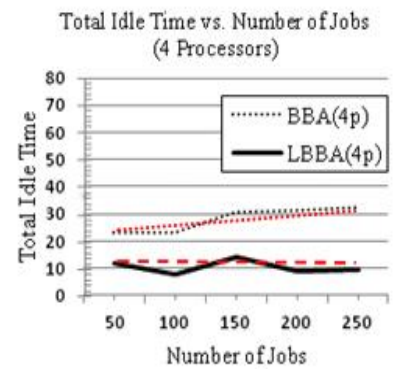

c)

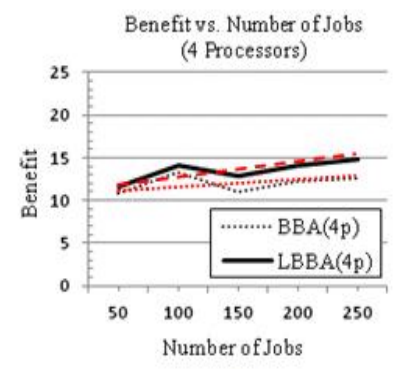

g)

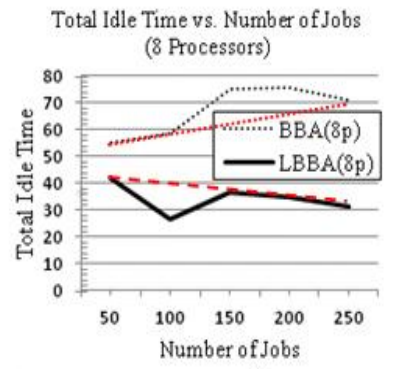

d)

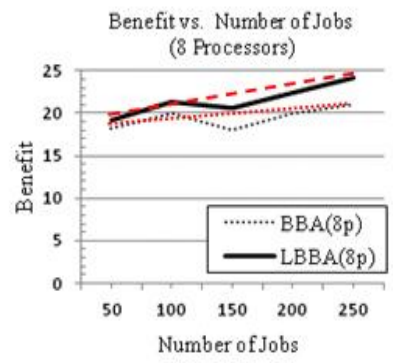

h)

Fig. 4. Comparison of total idle time and total benefit vs. number of the jobs on 2, 3, 4 and 8 processor system

The improvement (or increase) in the total benefit gained by LBBA ranged from $4 \%$ to $16.73 \%$ for a 4 processor system, and from $4.6 \%$ to $16.35 \%$ for an 8 processor system. As Fig. 4(e., f., g. and h.) illustrates, the more jobs we had in the system, the greater was the improvement in maximizing benefits by LBBA comparing to BBA.

The improvement (or decrease) of total processor idle time obtained by LBBA ranged from $44.21 \%$ to $69.18 \%$ for a 2 processor system, from $36.82 \%$ to $70.68 \%$ for a 3 processor system (Figure 4. a and $4 . b$ ), from $39.18 \%$ to $72.2 \%$ for a 4 processor system, and from $27.92 \%$ to $57.83 \%$ for 8 processors (Figure 4.c and 4.d). These results prove that LBBA consumes CPU cycles more conservatively.

The graphs show the actual data gathered through our extensive experiments, which is not normalized. The arrival times and computation times are randomly generated and job sets can consist of both synchronous and asynchronous jobs. These factors affect the workload of the system, which itself has a direct impact on the total benefit gained, number of completed jobs and total processor idle time. We intentionally consider a variety of possible scenarios to study how both algorithms perform in each situation. The red lines indicate the trends of total benefit and total idle time of the processors.

Despite of a little fluctuation, both BBA and LBBA have increasing trend in total benefit as the number of jobs in the system increases. Having fewer jobs in a period of time and heavier workload during another period would result in more processor idle time (while fewer jobs are in the system) and less benefit gained by the completed jobs that had to wait longer in the system during the heavier period. This can be the reason of the fluctuation of the graph for 100 and 150 jobs. BBA has an increasing trend of total idle time with increasing number of jobs in the system, even with more processors. However, total idle time in LBBA stays almost the same and considerably less than BBA. It also decreases in an 8 processor system and the difference of the trends is substantial in this case.

The improvement in both total gained benefit and benefit-to-cost ratio (Tables 3 and 4) by our algorithm show that the LBBA method can gain more benefit at lower cost, i.e., shorter makespan. Also, substantial decrease in total processor idle time by LBBA is a proof of its better resource management and CPU cycles usage.

However, one may argue that even though LBBA has much lower total idle time, it might have been using the processors to work on some jobs that are sent to garbage collection and their processing time is wasted not gaining any benefit while BBA was idle not starting those jobs. Our results (provided in Tables 5 and 6) prove that it is not the case. Table 5 provides the average ratio of missed deadlines for both algorithms. It shows that in all experiment settings, LBBA either had completed all the jobs, or if not, its miss ratio was lower than BBA for the same experiment. Therefore, we were able to gain more benefit in shorter makespan, with less miss ratio and more balanced usage of processors resulting in lower total idle time.

In addition, we observed a significant improvement in the scheduling speed by our proposed method (Table 6). For example, the simulation of the previous method took 16 minutes ( 960 seconds) on average to schedule sets 
of 250 jobs on 4 processors, while the average simulation time of our scheduling method was about 5 seconds for the same setting. The different in the speed of the methods get more significant for larger job sets. This is while the same design and implementation methods were used for both algorithms.

\begin{tabular}{|c|c|c|c|}
\hline No. of Jobs & BBA (2p) & LBBA (2p) & Improvement \\
\hline $\mathbf{2 0}$ & 7.29 & 8.06 & $10.62 \%$ \\
\hline $\mathbf{4 0}$ & 3.68 & 3.99 & $8.53 \%$ \\
\hline $\mathbf{6 0}$ & 2.96 & 3.18 & $7.32 \%$ \\
\hline $\mathbf{8 0}$ & 2.50 & 2.69 & $7.67 \%$ \\
\hline $\mathbf{1 0 0}$ & 2.05 & 2.19 & $6.86 \%$ \\
\hline
\end{tabular}

Table 3: Benefit-to-Cost Ratio and the Improvement Achieved by LBBA on a 2 Processor System

\begin{tabular}{|c|c|c|c|}
\hline No. of Jobs & BBA (3p) & LBBA (3p) & Improvement \\
\hline $\mathbf{2 0}$ & 13.40 & 14.40 & $7.45 \%$ \\
\hline $\mathbf{4 0}$ & 6.87 & 7.47 & $8.77 \%$ \\
\hline $\mathbf{6 0}$ & 5.60 & 6.08 & $8.40 \%$ \\
\hline $\mathbf{8 0}$ & 4.81 & 5.25 & $9.13 \%$ \\
\hline $\mathbf{1 0 0}$ & 4.03 & 4.35 & $7.91 \%$ \\
\hline
\end{tabular}

Table 4: Benefit-to-Cost Ratio and the Improvement Achieved by LBBA on a 3 Processor System

\begin{tabular}{|c|c|c|c|c|}
\hline No. of Jobs & BBA (4p) & LBBA (4p) & BBA (8p) & LBBA (8p) \\
\hline $\mathbf{5 0}$ & 0.084 & 0.00 & 0.00 & 0.00 \\
\hline $\mathbf{1 0 0}$ & 0.100 & 0.05 & 0.00 & 0.00 \\
\hline $\mathbf{1 5 0}$ & 0.067 & 0.00 & 0.00 & 0.00 \\
\hline $\mathbf{2 0 0}$ & 0.075 & 0.00 & 0.05 & 0.00 \\
\hline $\mathbf{2 5 0}$ & 0.100 & 0.00 & 0.02 & 0.00 \\
\hline
\end{tabular}

Table 5: Tardiness or Missed Deadline Ratio (\%)

\begin{tabular}{|c|c|c|c|c|}
\hline \multirow{2}{*}{ Number of Jobs } & \multicolumn{4}{|c|}{ Average schedulers' run time (in seconds): } \\
& BBA (4p) & LBBA (4p) & BBA (8p) & LBBA (8p) \\
\hline $\mathbf{5 0}$ & 2.50 & 1.00 & 2.00 & 0.50 \\
\hline $\mathbf{1 0 0}$ & 12.50 & 1.00 & 9.60 & 1.00 \\
\hline $\mathbf{1 5 0}$ & 120.00 & 2.00 & 60.00 & 1.00 \\
\hline $\mathbf{2 0 0}$ & 210.00 & 3.00 & 180.00 & 2.00 \\
\hline $\mathbf{2 5 0}$ & 960.00 & 5.00 & 870.00 & 3.00 \\
\hline
\end{tabular}

Table 6: Average schedulers' run time (in seconds)

\section{CONCLUSIONS AND FUTURE WORK}

In this paper we proposed, simulated and analyzed an efficient preemptive benefit-aware technique that prioritizes the jobs using their benefit density functions and schedules them in a real-time basis in order to maximize the total benefit gained by the completed jobs. In the meanwhile, it reduces the cost (makespan or overall flow time) of the jobs and total idle time of the processors by online choice of Greedy or load balancing approximations to partition jobs among multiple processors at the time of release.

We proposed an online choice of two approximation algorithms (Greedy and load balancing) as a solution for special cases that were not considered in the existing benefit-aware multiprocessor scheduling algorithms. Examples of those cases are when there are several jobs with the same priority or when a high priority job can be executed by more than one non-idle processor because it can preempt the running jobs on the top of their stacks. 
The results of our analysis and experiments show that our solution (LBBA) has improved the performance of the previous benefit-based method (BBA) explained in [29] while preserving its constant competitive ratio $\left(10 C^{2}\right)$. Our extensive experiments showed up to $72 \%$ improvement in total processor idle time, and improving the benefit awareness by up to $17 \%$ comparing to BBA. LBBA showed more improvement with heavier work load. This improvement is provided by more balanced distribution of jobs among processors and shorter flow times which will increase the total benefit. Also, a better resource management (CPU cycles in this case) is possible using our method. This advantage is beneficial to many real-time applications specially those that are running on batteryoperated computing devices.

In addition, we proved both analytically and by simulation that our solution is computationally less expensive so that the average run time of our LBBA scheduling algorithm worked up to about $290 \%$ faster than BBA (3 seconds for scheduling 250 jobs on an 8 processor system compared to 870 seconds taken by BBA). This advantage along with lower ratio of missed deadlines makes LBBA a considerably faster scheduling algorithm for multiprocessor systems comparing to the previous algorithm (BBA) which can improve the QoS (Quality of Service) for many real-time applications such as bursty hosting servers, video games and other multimedia systems. Another advantage of our new method is that no synchronization is needed for fetching the jobs from the pool. That is because a separate pool is assigned to each processor in contrast to the other method (BBA) where all processors use a shared pool.

We can summarize the advantages of LBBA as follows:

- LBBA is a novel hybrid model of soft real-time multiprocessor scheduling. Despite of BBA, which only follows benefit model, LBBA is a combination of benefit model and cost model. That is, it aims to minimize makespan in order to achieve the maximum benefit at the lowest cost.

- Reducing multi-processor scheduling into uniprocessor scheduling: BBA uses one pool shared by all the processors to keep the ready jobs while they're not scheduled. Hence, partitioning of each job is done when it is scheduled, and starts running on the assigned processor. Considering a separate pool for each processor, enables the LBBA scheduler to partition the jobs at their arrival time. The advantage of this method of early partitioning is that the NP-Hard problem of multiprocessor real-time scheduling will be reduced to a series of uniprocessor real-time scheduling.

- LBBA facilitates load-balanced partitioning of waiting jobs, while this case is not considered in BBA. For example, in case the waiting (or ready) jobs arrive asynchronously, then LBBA adapt the "Greedy Approximation" to assign a job to the pool of the processor with the least remaining workload. If jobs are synchronous, i.e. arrive at the same time, those that cannot start running and have to wait in a pool, will be partitioned among the processors, using our "Load-Balancing" technique.

- LBBA optimizes the CPU usage and minimizes the total idle time of the processors by balancing the workload among them.

- Improved QoS by reducing missed deadline ratio: As shown by an example in the manuscript, LBBA reduces the possibility of starvation for low priority jobs, comparing to BBA.

- LBBA is computationally less expensive than BBA, as proved in the paper.

- Minimal Response time: The overall Response Time, including both scheduling and execution time, for a job set in LBBA is tremendously shorter than in BBA for the same set. The heavier the workload of the system, the greater is the reduction of response time (up to $300 \%$ in our experiments).

In our future work, we continue our analysis to provide a clean cut complexity bound, and response time bound for LBBA. We also plan to use our method to schedule periodic job sets to explore its impact on the benefit, cost, total idle time and tardiness in those cases. 


\section{ACKNOWLEDGMENT}

This work is supported in part by the National Science Foundation under Awards No. 0720856 and No. 1219082.

\section{REFERENCES}

[1] E.G. Coffman, Jr., M.R. Garey, D.S. Johnson, Edited by D.S. Hochbaum, "Approximation Algorithms for NP-Hard Problems," Chapter 2: "Approximation Algorithms for Bin Packing: A Survey," page 67, 1997.

[2] R.L. Graham, "Bounds on Multiprocessing Timing Anomalies", SIAM Journal on Applied Mathematics, 17:263-269, 1969.

[3] L. Welch and S. Brandt, "Toward a Realization of the Value of Benefit in Real-Time Systems," Workshop on Parallel and Distributed Real-Time Systems (WPDRTS 2001), San Francisco, California, April 23-24, 2001.

[4] R. Rajkumar, C. Lee, J.P. Lehozcky, and D.P. Siewiorek, "A Resource Allocation Model for QoS Management," Proc. 18th IEEE Real-Time Systems Symposium, pp. 298-307, Dec. 1997.

[5] E. Chang and A. Zakhor, "Scalable Video Coding Using 3-D Subband Velocity Coding and Multi-Rate Quantization," Proc. IEEE Int'l Conf. Acoustics, Speech, and Signal Processing, pp. 574-577, July 1993.

[6] E. Chang and A. Zakhor, "Scalable Video Data Placement on Parallel Disk Data Arrays," Proc. ISIT/SPIE Symp. Electronic Imaging Science and Technology, pp. 208-223, Feb. 1994.

[7] W. Feng and J.W.S. Liu, "An Extended Imprecise Computation Model for Time-Constrained Speech Processing and Generation," Proc. IEEE Workshop Real-Time Applications, pp. 76-80, May 1993.

[8] C.J. Turner and L.L. Peterson, "Image Transfer: An End-to-End Design," Proc. SIGCOMM Symp. Comm. Architectures and Protocols, pp. 258-268, Aug. 1992.

[9] M. Boddy and T. Dean, "Solving Time-Dependent Planning Problems," Proc. 11th Int'l Joint Conf. Artificial Intelligence (IJCAI-89), pp. 979-984, Aug. 1989.

[10] B. Hayes-Roth, "Architectural Foundations for Real-Time Performance in Intelligent Agents," Journal of Real-Time Systems, vol. 2, no. 1, pp. 99-125, 1990.

[11] S. Zilberstein and S.J. Russell, "Anytime Sensing, Planning and Action: A Practical Model for Robot Control," Proc. 13th Int'l Joint Confs. Artificial Intelligence, pp. 1402-1407, 1993.

[12] E.J. Horvitz, "Reasoning under Varying and Uncertain Resource Constraints," Proc. Seventh Nat'l Conf. Artificial Intelligence (AAAI-88), pp. 111-116, Aug. 1988.

[13] J. Grass and S. Zilberstein, "A Value-Driven System for Autonomous Information Gathering," Journal of Intelligent Information Systems, vol. 14, pp. 5-27, Mar. 2000.

[14] R.E. Korf, "Real-Time Heuristic Search," Artificial Intelligence, vol. 42, no. 2, pp. 189-212, 1990.

[15] S.V. Vrbsky and J.W.S. Liu, "APPROXIMATEĐA Query Processor that Produces Monotonically Improving Approximate Answers," IEEE Trans. Knowledge and Data Eng., vol. 5, no. 6, pp. 1056-1068, Dec. 1993.

[16] Jayavardhana Gubbi, Rajkumar Buyya , Slaven Marusic , Marimuthu Palaniswami, "Internet of Things (IoT): A vision, architectural elements, and future directions", Future Generation Computer Systems 29 (2013) 16451660

[17] S. Irani and Anna R. Karlin, edited by D.S. Hochbaum, "Approximation Algorithms for NP-Hard Problems," Chapter 13: "Online Computation," page 552, 1997.

[18] C.Y. Yang, J. Chen, and T.W. Kuo, "An Approximation Algorithm for Energy-Efficient Scheduling on A Chip Multiprocessor," ACM/IEEE Design, Automation, and Test in Europe (DATE), Munich, Germany, March 2005.

[19] J. J. Chen, T.W. Kuo, and C.Y. Yang, "Real-Time Task Replication for Fault Tolerance in Identical Multiprocessor Systems," the 13th IEEE Real-Time and Embedded Technology and Applications Symposium (RTAS), Bellevue, WA, USA, April 3-6, 2007.

[20] B. Sanati and A.M.K. Cheng, "Maximizing Job Benefits on Multiprocessor Systems Using a Greedy Algorithm," WiP session of the 14th IEEE Real-Time and Embedded Technology and Applications Symposium (RTAS), April, 2008

[21] B. Sanati and A.M.K. Cheng, "Efficient Online Benefit-Aware Multiprocessor Scheduling Using an Online Choice of Approximation Algorithms," the 11th IEEE International Conference on Embedded Software and Systems (ICESS 2014), Paris, France, August 20-22, 2014

[22] C.F. Kuo, T.W. Yang, and T.W. Kuo, "Dynamic Load Balancing for Multiple Processors," IEEE 12th International Conference on Real-Time and Embedded Computing Systems and Applications (RTCSA 2006), Sydney, Australia, August 16-18, 2006.

[23] E. Piel, P. Marquet, J. Soula and J.L. Dekeyser, "Load-Balancing for a Real-Time System Based on Asymmetric Multi-Processing," The $16^{\text {th }}$ Euromicro Conference on RealTime Systems, WIP, April 2004.

[24] S.A. Brandt and G.J. Nutt, "Flexible Soft Real-Time Processing in Middleware," Journal of Real- Time 
Systems, Kluwer, 2001.

[25] E. D. Jensen, C.D. Locke and H. Tokuda, "A Time Driven Scheduling Model for Real-Time Operating Systems," Proceedings of the Real-Time Systems Symposium, 112-122, IEEE CS Press, 1985.

[26] L.R. Welch, B. Ravindran, B. Shirazi and C. Bruggeman, "Specification and Analysis of Dynamic, Distributed Real-Time Systems," in Proceedings of the 19th IEEE Real-Time Systems Symposium, 72-81, IEEE Computer Society Press, 1998.

[27] D. Andrews, L.R. Welch, and S. Brandt, “A Framework for Using Benefit Functions In Complex Real Time Systems", Journal of Parallel and Distributed Computing Practices, Volume 5, No. 1, March 2002.

[28] G. Buttazzo, M. Spuri and F. Sensini, "Value vs. Deadline Scheduling in Overload Conditions," in Proceedings of the 19th IEEE Real-Time Systems Symposium, IEEE Computer Society Press, 1998.

[29] B. Awerbuch, Y. Azar, and O. Regev, "Maximizing Job Benefits On-Line", Proceedings of the third International Workshop, APPROX, Germany, September 2000.

[30] H. Aydin, R. Melhem, D. Mosse and P. Mejia-Alvarez, "Optimal Reward-Based Scheduling For Periodic Real-Time Tasks," IEEE Transactions On Computers, Vol. 50, No. 2, February 2001.

[31] J.K. Dey, J. Kurose, and D. Towsley, “On-Line Scheduling Policies for a Class of IRIS (Increasing Reward with Increasing Service) Real-Time Tasks,” IEEE Trans. Computers, vol. 45, no. 7, pp. 802-813, July 1996. 